\title{
Angiosarcoma pulmonar metastásico
}

\author{
M. GARCÍA CLEMENTE, T. GONZÁLEZ BUDIÑO, J. ESCOBAR STEIN ${ }^{1}$, \\ A. J. SECO GARCÍA, C. CELORIO PEINADO ${ }^{2}$, J. RODRÍGUEZ RODRÍGUEZ \\ Sección de Neumología. ${ }^{2}$ Servicio de Radiología. Hospital Álvarez-Buylla. Mieres.

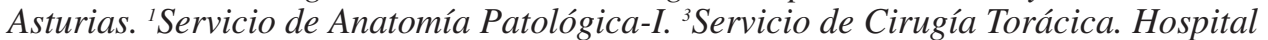 \\ Central de Asturias
}

\author{
METASTATIC ANGIOSARCOMA OF THE LUNG
}

\begin{abstract}
RESUMEN
La afectación pulmonar por angiosarcoma, habitualmente en forma de enfermedad metastásica, es una rara enfermedad. El angiosarcoma pulmonar primario es un tumor extremadamente raro. El pulmón es uno de los lugares más frecuentes de afectación metastásica junto con el hígado y los ganglios linfáticos. La hemoptisis es el síntomas más frecuente, pudiendo presentarse incluso en forma de hemorragia pulmonar difusa. La radiografía de tórax puede ser normal, pero lo más frecuente es la presencia de múltiples imágenes nodulares. No es habitual que se establezca un diagnóstico precoz por el bajo índice de sospecha derivado de la rareza del tumor. El tratamiento sigue siendo desalentador sobre todo en los angiosarcomas metastásicos cuya supervivencia media es de 9 meses tras el diagnóstico.
\end{abstract}

PALABRAS CLAVE: Angiosarcoma pulmonar. Tumor vascular.

\begin{abstract}
Angiosarcoma involving the lung, usually a result of metastatic disease, is a rare disorder. Primary angiosarcoma of the lung is an extremely rare tumor. The lung is one of the most common sites of metastatic involvement, along with liver and lymph nodes. Hemoptysis is the most frequent presenting symptom even with diffuse pulmonary hemorrhage. Chest radiography may reveal a spectrum of findings ranging form normal to bilateral nodular lesions. Early diagnosis is not common because of the rarity of angiosarcoma in the lung and hence, low index of suspicion. The prognosis is generally poor, with a median survive of 9 months after diagnosis.
\end{abstract}

KEY WORDS: Angiosarcoma pulmonar. Tumor vascular.

García Clemente M, González Budiño T, Escobar Stein J, Seco García AJ, Celorio Peinado C, Rodriguez Rodríguez J. Angiosarcoma pulmonar metastásico. An Med Interna (Madrid) 2004; 21: 27-30.

\section{INTRODUCCIÓN}

El angiosarcoma es un tumor vascular maligno poco frecuente (1 al $2 \%$ del total de sarcomas). La afectación pulmonar por este tumor no es habitual y suele ser debida a metástasis de un tumor primario de otro origen. El diagnóstico de angiosarcoma pulmonar primario es extremadamente raro (1). Dado que la mayor parte de las publicaciones son casos clínicos aislados, las manifestaciones y el curso clínico no son bien conocidos. Presentamos un caso de angiosarcoma pulmonar metastásico por su interés y rareza.

\section{CASO APORTADO}

Paciente de 58 años que acudió al servicio de urgencias por hemoptisis de 5 días de evolución. Se trataba de una mujer, no fumadora, con diabetes tipo II controlada con dieta. Padecía desde hacía varios años un síndrome depresivo en tratamiento actualmente con venlafaxina $75 \mathrm{mg}$ al día y alprazolam $0,5 \mathrm{mgr}$ cada 24 horas. Hacía 4 y 2 años había presentado sendos episodios de trombosis venosa profunda. Acudió a urgencias en esta ocasión por notar en los últimos 5 días disnea y expectoración hemoptoica de sangre roja acompañada de coágulos, dolor torácico en hemitórax izquierdo no claramente definido y sensación distérmica. No síndrome general acompañante. En el examen físico no había datos destacables salvo febrícula de $37,8^{\circ} \mathrm{C}$ y discreta taquicardia sinusal (104 /minuto). En los exámenes complementarios el hemograma, el estudio de coagulación, la bioquímica hepática y el sistemático y sedimento de orina fueron normales. En la bioquímica general solo llamaba la atención una glucemia de $121 \mathrm{mg} / \mathrm{dl}$. La gasometría arterial al ingreso mostraba $\mathrm{PaO}_{2}$ de $69 \mathrm{~mm}$ de $\mathrm{Hg}, \mathrm{PaCO}_{2}$ de 45 $\mathrm{mm}$ de $\mathrm{Hg}$, $\mathrm{pH}$ de 7,39 y bicarbonato de $25,6 \mathrm{mmol} / \mathrm{l}$. En la radiografía de tórax, se observaron imágenes nodulares bilaterales difusas de diferentes tamaños y en la tomografía axial computerizada

Trabajo aceptado: 24 de julio de 2003

Correspondencia: Marta García Clemente. Sección de Neumología. Hospital Álvarez-Buylla. Murias, s/n. 33600 Mieres, Asturias. e-mail: mgclemen@las.es 
(TAC) de tórax múltiples lesiones nodulares, fundamentalmente en campos superiores y medios de ambos pulmones, de localización periférica y bordes imprecisos, no observándose adenopatías mediastínicas (Fig. 1). La TAC de abdomen fue normal. Los hemocultivos y las serologías para gérmenes productores de neumonía atípica, la serología de VIH y las baciloscopias y citologías de esputo fueron negativos. Al ingreso se pautó tratamiento con amoxicilina-clavulánico a dosis de $1 \mathrm{~g}$ intravenoso cada 8 horas. Ante la persistencia de hemoptisis, a las 48 horas del ingreso se realizó broncoscopia que puso de manifiesto la presencia de sangre con coágulos en tráquea y árbol bronquial bilateral sin observarse lesiones endobronquiales. La baciloscopia y las citologías de aspirado bronquial fueron negativas. Se realizaron determinaciones de anticuerpos antinucleares, anti-ADN, anticitoplasma del neutrófilo (ANCA), antimembrama basal glomerular, anticardiolipina, células $\mathrm{LE}$, factor reumatoideo y complemento que fueron negativos. Al séptimo día comenzó con fiebre elevada de $38-39^{\circ} \mathrm{C}$ y dolor en la pared torácica anterior que aumentaba con los movimientos respiratorios e incremento de la hemoptisis. Se objetivó en el examen físico la aparición de una fibrilación auricular con respuesta ventricular rápida a 140 latidos por minuto. La radiografía de tórax puso de manifiesto un aumento de tamaño de la silueta cardiaca y empeoramiento de las imágenes nodulares con infiltrados alveolares bilaterales acompañantes. Se realizó ecografía cardiaca observándose derrame pericárdico moderado sin evidencia de taponamiento ni signos de endocarditis. Se digitalizó a la paciente y se inició tratamiento con rifampicina, isoniacida y pirazinamida de forma empírica. Se realizó punción pulmonar siendo tanto los cultivos como las citologías del material extraido negativos. Al décimo día ante el empeoramiento clínico y gasométrico de la paciente (gasometría arterial con $\mathrm{FiO}_{2}$ al $35 \%: \mathrm{PaO}_{2}: 52, \mathrm{PaCO}_{2}: 35, \mathrm{pH}: 7,38$ y bicarbonato: $27,8 \mathrm{mmol} / \mathrm{l}$ ) se añadió 6-metilprednisolona a dosis de $80 \mathrm{mg}$ al día con desaparición de la fiebre y ligera mejoría de la insuficiencia respiratoria. En el control ecocardiográfico se observó aumento de tamaño del derrame pericárdico y en la radiografía de tórax aumento de los infiltrados alveolares bilaterales por lo que se solicitó al servicio de Cirugía Torácica la realización de biopsia pulmonar siendo el diagnóstico compatible con angiosarcoma pulmonar metastásico de origen no especificado (Fig. 2). La paciente fue trasladada al servicio de Oncología médica donde falleció a las 24 horas de iniciar el tratamiento con quimioterapia. La autopsia no fue concedida.

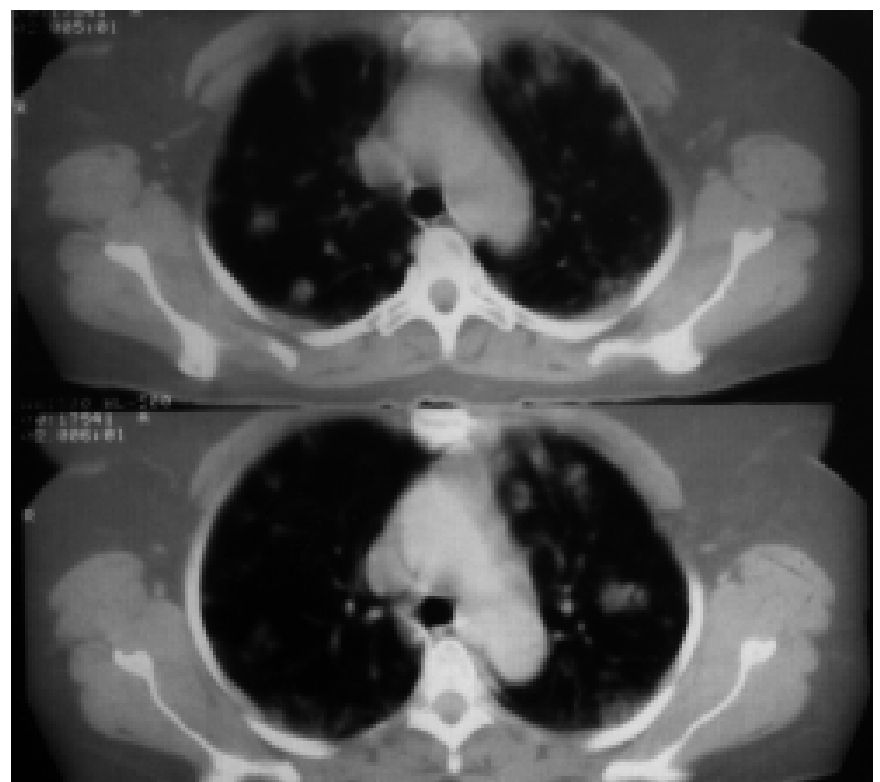

Fig. 1. TAC de tórax con imágenes nodulares bilaterales de bordes imprecisos.

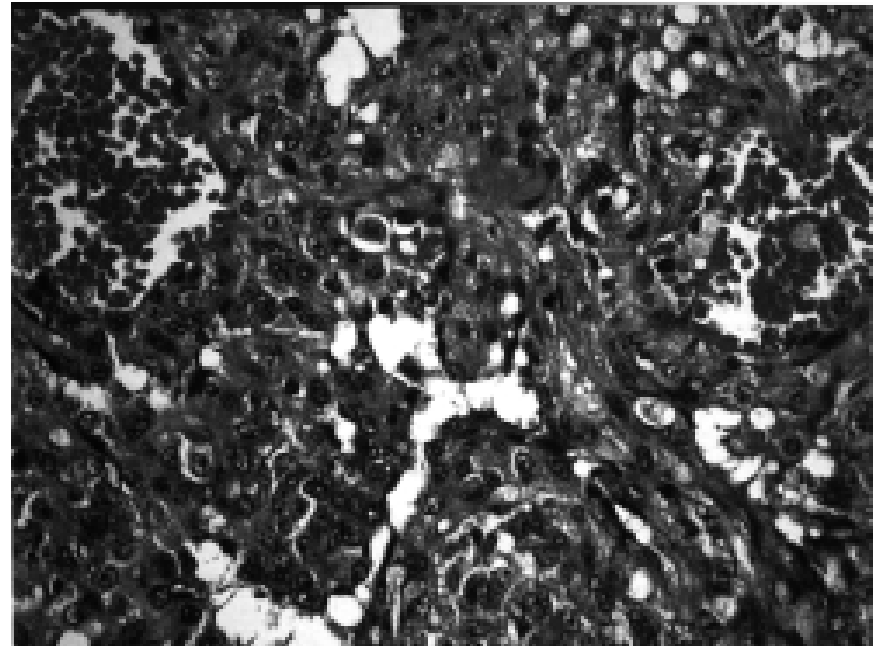

Fig. 2. Biopsia pulmonar con tinición de hematoxilina-eosina en la que se observa una lesión con vasos de contornos irregulares, que presenta endotelios de células malignas, pleomórficas que se proyectan hacia las luces. Las células tumorales también forman áreas sólidas con frecuentes mitosis.

\section{DISCUSIÓN}

El angiosarcoma es un tumor vascular maligno poco frecuente que afecta predominantemente a varones con mayor incidencia a partir de los 60 años. Cualquier órgano puede ser el origen primario de este tumor siendo los lugares más frecuentes de afectación la piel (33\%) y tejidos blandos profundos $(24 \%)$. Les siguen a distancia, la mama (8\%), hígado $(8 \%)$, hueso $(6 \%)$, bazo $(4 \%)$, corazón y grandes vasos $(3 \%)$ órbita (3\%), área ORL (4\%) y otros $(7 \%)(2,3)$. La afectación pulmonar por angiosarcoma es rara, siendo más frecuente la afectación metastásica que la presencia de un tumor primario pulmonar, aunque desde el punto de vista clínicopatológico son superponibles ambas formas (4). El pulmón es uno de los lugares más frecuentes de afectación metastásica junto con el hígado y los ganglios linfáticos, apareciendo metástasis pulmonares en el 60-80\% de los angiosarcomas cutáneos y cardí$\operatorname{acos}(1)$.

En lo que se refiere a la etiología, se ha relacionado el angiosarcoma hepático con la exposición a cloruro de vinilo, pesticidas derivados del arsénico y dióxido de torio (Thorotrast) $(5,6)$. También se han descrito casos de angiosarcomas óseos y de tejidos blandos tras radiaciones (5) y casos postmastectomía radical (con aparición del tumor en la extremidad superior con linfedema)(1). Se ha publicado por último relación entre el uso de feniletilhidracina y el desarrollo de angiosarcoma (7).

Clínicamente destaca en casi todos los casos la presencia de hemoptisis, que puede ser episódica con intervalos libres probablemente por hemorragias autolimitadas, como ocurrió en nuestra paciente o en forma de hemoptisis masiva (8). Patel y Ryu (1) en su serie objetivaron hemoptisis en el 50\% de los pacientes. Otra sintomatología frecuente es la pérdida de peso, tos, dolor torácico o disnea. A veces se presentan con clínica atribuible a la afección pleural, como neumotórax, hemoneumotórax o derrame pleural en relación con metástasis subpleurales, o incluso con cavitación de las lesiones $(1,9)$. En 
ocasiones aparecen en forma de hemorragia pulmonar difusa $(4,7,10-12)$ a la que pueden atribuirse los infiltrados alveolares visualizados en nuestra paciente en la evolución de la enfermedad y la insuficiencia respiratoria que presentó en los momentos finales. Hasta un $20 \%$ de los casos son asintomáticos y sólo se descubren en la autopsia (1). Yousem (4) y Zwaveling et al (8), presentan casos similares al nuestro, observándose en el caso de este último autor hemoptisis, infiltrados nodulares bilaterales y anemia, lo que llevó en principio a hacer el diagnóstico erróneo de hemosiderosis pulmonar idiopática.

En nuestro caso, al igual que en los casos de Juan Pastor y Riu (2) y Bic et al (5) la afectación pulmonar y pericárdica hace pensar que el tumor primario pudiera estar situado en órganos próximos a estas dos localizaciones y con alto potencial de metastatización. En este sentido, debe de considerarse la posibilidad de angiosarcoma primario cardíaco como mayor probabilidad, y ya menos probable el angiosarcoma primario de arteria pulmonar y el angiosarcoma primario pericárdico; los tumores en estas localizaciones metastatizan frecuentemente al pulmón y no es infrecuente que se manifiesten clínicamente sólo como enfermedad pulmonar. El angiosarcoma cardíaco es una enfermedad rara, de mal pronóstico, que aparece preferentemente en varones y en la edad media de la vida (5). En la serie de Strohl (13), el $86 \%$ de los pacientes se presentaron con síntomas de enfermedad pericárdica o fallo cardíaco derecho congestivo causado por una obstrucción de la vena cava u obstrucción del tracto de salida del ventrículo derecho. La aurícula derecha es con mucho el lugar más frecuente de origen de la enfermedad. Se pueden observar metástasis en los pulmones, ganglios linfáticos e hígado (13).

Es difícil hacer el diagnóstico de angiosarcoma cardíaco antes de la necropsia (8). En el caso descrito por Bic et al. (5) el paciente cursó con derrame pericárdico como ocurrió en nuestro caso, sin embargo pese a haber realizado tres ecografías cardíacas y revisadas éstas después de obtener el diagnóstico en la necropsia, no se pudo evidenciar en las mismas la presencia del tumor cardíaco. No existe una interpretación para explicar esta discrepancia aunque algunos factores como la ecogenicidad atípica y el desarrollo concéntrico del tumor pueden contribuir a ello. La RNM y la ecocardiografía transesofágica parecen ser más prometedoras por su mayor sensibilidad y capacidad resolutiva $(5,14)$.

La afectación pericárdica por angiosarcoma también es muy infrecuente y muchas veces representa la extensión pericárdica de un angiosarcoma cardíaco primario (2). Suele ser más frecuente en varones y la afectación pericárdica es habitualmente difusa. Clínicamente se presentan como derrame pleuropericárdico hemorrágico, fiebre y dolor torácico, y no es infrecuente que se orienten como un proceso infeccioso o inflamatorio. Acostumbra a tener una evolución rápida con constricción o taponamiento cardíaco y tiene una especial tendencia a producir metástasis pulmonares.

En los grandes vasos torácicos como la arteria pulmonar, la aorta o incluso las venas pulmonares, se dan sarcomas primarios y entre los diferentes tipos se encuentra el angiosarco- ma. Son tumores que afectan más a mujeres, sobre todo a partir de los 40 años de edad. Clínicamente destaca siempre la afectación pulmonar, pues metastatizan en el $70 \%$ de los casos. En la arteria pulmonar se dan sobre todo en el tronco principal y no es infrecuente la afectación pericárdica por extensión transmural del tumor (2).

El diagnóstico precoz no es habitual por el bajo índice de sospecha derivado de la rareza del tumor. La radiografía de tórax puede revelar un gran número de hallazgos que van desde la radiografía de tórax normal hasta la presencia de múltiples imágenes nodulares que es la forma de presentación en el $70 \%$ de los casos $(1,2)$ con o sin derrame pleural acompañante, hasta infiltrados alveolares difusos compatibles con hemorragia pulmonar, habiéndose presentado en nuestra paciente ambas formas radiológicas a lo largo de la evolución de la enfermedad. Puede presentarse también como una lesión única. El papel de la ecocardiografía, el TAC y la RNM no está claro en esta rara entidad, pero parece que la RNM del tórax puede ayudar a confirmar la naturaleza vascular del angiosarcoma. Tanto la biopsia transbronquial como la biopsia transtorácica se han utilizado para establecer el diagnóstico sin riesgos de sangrado. En muchos casos se precisa una biopsia pulmonar abierta para llegar al diagnóstico, como ocurrió en nuestra paciente $(10)$.

Aunque los criterios histopatológicos iniciales para el diagnóstico de angiosarcoma fueron descritos por Stout en 1940, la distinción entre lesiones benignas y malignas así como la definición del lugar primario de la neoplasia pueden ser difíciles. Además pueden encontrarse hallazgos histopatológicos similares en el tumor bronquioloalveolar intravascular, el sarcoma de Kaposi y otros tumores vasculares menos diferenciados (1).

El tratamiento sigue siendo desalentador sobre todo en los angiosarcomas metastásicos $(5,7,8)$ con una supervivencia media de 9 meses tras el diagnóstico (1). Se han utilizado diferentes modalidades terapéuticas como radioterapia, quimioterapia (incluyendo adriamicina y metrotexate), e intervenciones quirúrgicas como la resección en cuña o la neumonectomía pero ninguna ha demostrado ser dramáticamente efectiva. El pronóstico puede estar influenciado por el grado de diferenciación del tumor, siendo las neoplasias bien diferenciadas de un curso más indolente.

En nuestro caso la infiltración difusa de ambos pulmones por el tumor, asociado al sangrado alveolar, condujo a la paciente a una insuficiencia respiratoria. Al igual que en el caso de Segal et al (10), la paciente evolucionó fatalmente en un corto espacio de tiempo desde el inicio de los síntomas.

Es importante incluir el angiosarcoma en el diagnóstico diferencial de pacientes con hemoptisis e imágenes nodulares radiológicas. En aquellos pacientes que presenten de forma simultánea metástasis pulmonares y derrame pericárdico debe de sospecharse la posibilidad de un tumor cardíaco. Por último, en caso de objetivarse una hemorragia pulmonar en pacientes que hayan recibido radioterapia previa o en pacientes con una mastectomía radical debe de considerarse la posibilidad de un angiosarcoma primario o metastásico pulmonar $(1,10)$. 


\section{Bibliografía}

1. Patel AM, Ryu JH Angiosarcoma in the lung. Chest 1993; 103: 15311535.

2. Juan Pastor A, Riu F. Hemoptisis e infiltrados pulmonares en una mujer de 22 años. Med Clin 1999; 112:349-355.

3. Enzinger FM, Weiss SW. Soft tissue sarcoma, 4 th ed. St. Louis: Mosby, 2001:917-954.

4. Adem C, Aubry MC, Tazelaar HD, Myers JL. Metastatic angiosarcoma masquerading a diffuse pulmonary hemorrhage: clinicopathologic analysis of 7 new patients. Arch Pathol Lab Med 2001; 125: 1562-1565.

5. Bic JF, Fade-Schneller O, Marie B, Neimann JL, Anthoine D, Martinet Y. Cardiac angiosarcoma revealed by lung metastases. Eur Respir J 1994; 7: 1194-1196

6. Baxter PJ, Langlands AO, Anthony PP, Macsween RN, Scheuer PJ. Angiosarcoma of the liver: a marker tumor for the late effects of Thorotrast in Great Britain. Br J Cancer 1980; 41:446-453.

7. Spragg RG, Wolf PL, Haghighi P, Abraham JL, Astarita RW. Angiosarcoma of the lung with fatal pulmonary hemorrhage. Am J Med 1983; 74: 1072-1076

8. Zwaveling JH, Van Berkhout FT, Haneveld GT. Angiosarcoma of the heart presenting as pulmonary disease. Chest 1988; 94: 216-218.

9. Tateishi U, Hasegawa T, Kusumoto M, Yamazaki N, Iinuma G, Muramatsu Y, Moriyama N. Metastatic angiosarcoma of the lung: Spectrum of CT findings. Am J Roentgenol 2003; 180: 1671-1674.

10. Segal SL, Lenchner GS, Cichelli AV, Promisloff RA, Hofman WI, Baiocchi GA. Angiosarcoma presenting as diffuse alveolar hemorrhage. Chest 1988; 94: 214-216.

11. Pérez Marín JC, Apolinario Hidalgo RM, Suárez Cabrera M, Pena Quintana P, Cardenes Santana MA. Cutaneous angiosarcoma and bilateral pulmonary bleeding. An Med Interna (Madrid) 2002; 19: 607.

12. Bocklage T, Leslie K, Yousem S, Colby T. Extracutaneous angiosarcomas metastatic to the lungs: clinical and pathologic features of twenty one cases. Modern Pathology 2001; 14: 1216-1225.

13. Strohl KP. Angiosarcoma of the heart. Arch Intern Med 1976; 136:928929.

14. Freedberg RS, Kronzon I, Rumancik WM, Liebeskind D. The contribution of magnetic resonance imaging to the evaluation of intracardiac tumors diagnosed by echocardiography. Circulation 1988; 77: 96-103. 